\title{
Gravity on crystallisation of lysozyme: slower or faster?
}

Huaiyu Yang ${ }^{\alpha}$, Lei Huang ${ }^{\curlyvee}$, Fan Zhang ${ }^{\beta}$, Vikram Karde ${ }^{Y}$, Zhongqiang, Yang ${ }^{\S}$, Jerry YY Heng ${ }^{\gamma *}$

a Department of Chemical Engineering, Loughborough University, Loughborough, UK

$\checkmark$ Department of Chemical Engineering, Imperial College London, London, UK

$\beta$ Department of Chemical Engineering and Science, Royal Institute of Technology KTH, Stockholm, Sweden

${ }^{\S}$ Key Laboratory of Organic Optoelectronics \& Molecular Engineering of the Ministry of Education, Department of Chemistry, Tsinghua University, Beijing, China

\section{Supporting information}

Table 1 Gravity and rpm

\begin{tabular}{|l|l|}
\hline rpm & G \\
\hline 0 & 1 \\
\hline 500 & 27 \\
\hline 1000 & 106 \\
\hline 3000 & 956 \\
\hline 5000 & 2655 \\
\hline 7000 & 5204 \\
\hline 10000 & 10621 \\
\hline 14000 & 20817 \\
\hline
\end{tabular}




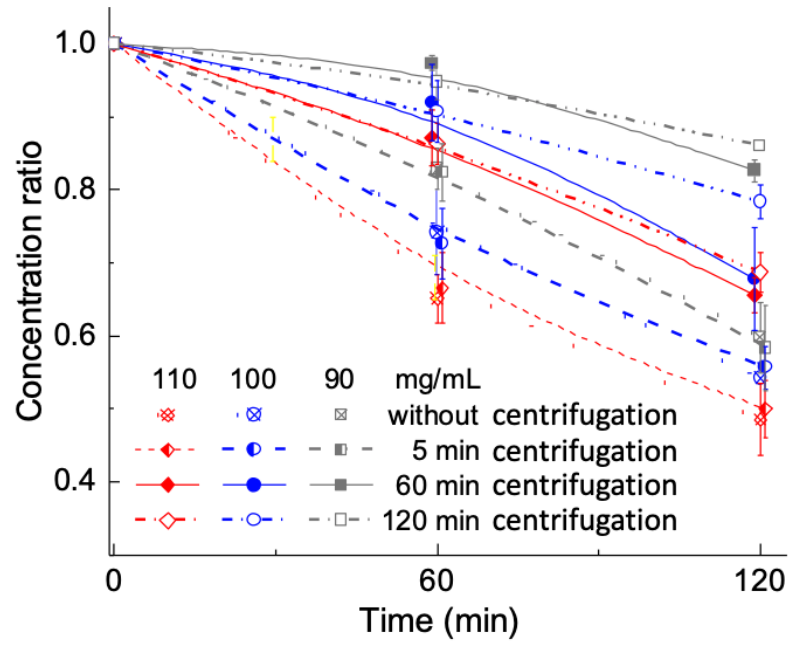

Figure 1 Concentrations of lysozyme with centrifugation at $7000 \mathrm{rpm}$ and centrifugation time $0-120 \mathrm{~min}$. 